\title{
Antimicrobial Profile and Phenotypic Metallo- $\beta$-Lactamase Detection of Acinetobacter baumannii Isolated From Clinical and Environmental Specimens
}

\author{
Himen Salimizand ${ }^{1}$; Farzan Modarresi ${ }^{2}$; Omid Azizi ${ }^{2,}$; Shahla Mansouri ${ }^{3}$; Mehdi Rahmati ${ }^{3}$; \\ Khabat Barkhordari ${ }^{3}$ \\ ${ }^{1}$ Department of Microbiology and Virology, Faculty of Medicine, Kurdistan University of Medical Sciences, Sanandaj, IR Iran \\ ${ }^{2}$ Department of Microbiology, Kerman Medical Students Research Committee, Kerman University of Medical Sciences, Kerman, IR Iran \\ ${ }^{3}$ Department of Microbiology and Immunology, Kerman University of Medical Sciences, Kerman, IR Iran \\ ${ }^{*}$ Corresponding author: Omid Azizi, Department of Microbiology, Kerman Medical Students Research Committee, Kerman University of Medical Sciences, Kerman, Iran. \\ E-mail: omid201262@gmail.com
}

Received: February 3, 2014; Accepted: April 22, 2014

\begin{abstract}
Background: One of the most challenging isolates in nosocomial infections is Acinetobacter baumannii which is rapidly changing towards multi-drug resistant. Resistance to the last resort antibiotics, carbapenems, is reported around the world. In particular, metallo- $\beta$ lactamases (MBL) are responsible and detection of MBLs is of interest.

Objectives: In this study we have evaluated the prevalence of MBLs in A. baumannii isolated during 2012 by two feasible described methods. Materials and Methods: In this cross sectional study, during 2012, 40 A. baumannii non-replicate isolates gathered from clinical and environmental specimens from Afzalipur hospital of Kerman. Isolates were characterized by conventional biochemical tests as $A$. baumannii-calcoaceticus complex (ABC). For antimicrobial susceptibility testing, 17 different antibiotics were sought by agar diffusion method. Phenotypic MBL detection was assessed by double disk test and Modified Hodge test for carbapenem resistant isolates.

Results: During the study 40 isolates of ABC were collected. Of which, 23 (57\%) isolates were resistant to carbapenems. Phenotypic MBLs detection was negative by two methods.

Conclusions: The rates of resistance of the isolated $\mathrm{ABC}$ in our clinical setting were at high level. Though, carbapenems were the most efficient antibiotic, but, there was a pronounced rate of resistant. MBL genes were not responsible for carbapenem resistance in under study clinical setting.
\end{abstract}

Keywords: Carbapenemases; Antimicrobial susceptibility; Acinetobacter baumannii

\section{Background}

One of the most important pathogens in nosocomial infections is Acinetobacter baumannii which is rapidly changing towards multi-drug resistant, especially in critical care units, in many regions around the world [1-3]. A. baumannii has isolated from different sources including urinary tract infections, surgical-site infections, septicemia, meningitis, ventilator associated pneumoniae and endocarditis [46]. Resistance to the last-resort antibiotic treatment, carbapenems, has reported more and more [7].

Carbapenem resistant $A$. baumannii (CRAB) is attributed to various reasons including produce oxacillinases (OXA) and metallo beta lactamases (MBLs), reduced expression of outer membrane proteins (29 kDa, 33-36 $\mathrm{kDa}$ ) and efficient efflux pumps [7, 8]. Metallo beta lactamases belong to Ambler class B and they differ from other carbapenemases in having broad substrate profile, potential for horizontal transfer and lack of inhibition by serine beta lactamases $[9,10]$. Besides, they require zinc ion as a metal cofactor for carbapenem activ- ity. This characteristic helps investigators for detecting MBL producer bacteria through ethylene-diamin-tetraacetic acid (EDTA) inhibition as co-factor inhibitor [11, 12]. Hospital laboratory needs rapid MBL detection tests, while encounter a resistant bacteria. Rapid detection methods are widely favorable especially in hospitals outbreaks. Therefore, they are of value to investigate in medical centers.

Although, polymerase chain reaction (PCR) technique is reliable and highly accurate, but instrument setting is costly and its accessibility is limited to the reference laboratories. Developing countries affected more in this issue. Another commercially available method, E-test (by AB Biodisk, Solna, Sweden) which consider imipenem in one side of strip and imipenem-EDTA in the other side is costly and insensitive in detecting most carbapenem susceptible MBL-harboring organisms, especially with $A$. baumannii isolates with MIC $\leq 4$ [13].

Recently, CLSI has published a method, Modified Hodge

Copyright (C) 2015, Zahedan University of Medical Sciences. This is an open-access article distributed under the terms of the Creative Commons Attribution-NonCommercial 4.0 International License (http://creativecommons.org/licenses/by-nc/4.0/) which permits copy and redistribute the material just in noncommercial usages, provided the original work is properly cited. 
test (MHT) in which carbapenemases producing Enterobacteriaceae detected. Nevertheless of supporting data from this institute for successful detection of carbapenemases in non-fermenting isolates such as Acinetobacter spp. and Pseudomonas spp., this method used for all carbapenemase producing isolates [11].

During 2011, there have been increasing reports of carbapenem resistant of A. baumannii isolates, from Afzalipur hospital, Kerman.

\section{Objectives}

Therefore, because of essentiality of detection and eradication of the source of reported isolates, in this study we have evaluated the prevalence of MBLs in A. baumannii isolated during 2012 by two feasible described methods.

\section{Materials and Methods}

Bacterial isolates: In this cross sectional study, $40 \mathrm{~A}$. baumannii non-replicate isolates gathered during a year, 2012, from Afzalipur hospital of Kerman. All specimens from all hospital wards and specimens from infection control program were included in the study. Characterizations of isolates were based on Gram negative staining and motility, routine biochemical reactions and negative oxidase activity.

Antibacterial susceptibility testing: All A. baumannii isolates were tested to 14 different antibiotics by Kirby-Bauer agar diffusion methods, in accordance with CLSI guidelines [11]. Antibiotic disks were purchased from MAST brand (MAST, UK) including imipenem, meropenem, ceftazidime, cefotaxime, ceftriaxone, cefepime, piperacillin, aztreonam, ampicillin-sulbactam, amikacin, tobramycin, gentamicin, ciprofloxacin and colistin. E. coli ATCC 25922 was tested as negative control.

Phenotypic detection of MBLs: To screen MBL producer A. baumannii isolates, imipenem and meropenem resistant isolates were considered for phenotypic detection by two methods. Previously described Modified Hodge test (MHT) [11] and double disk test (DDT) [12] together could cover most of prevalent MBL enzymes detection. In the former method which is based on the using of chelating agents, at first, isolates inoculated on Müller Hinton Agar (Merck, Germany). Then, an imipenem and blank disks (MAST, UK) placed on the surface by $10 \mathrm{~mm}$ (edge to edge). Blank disk moistened with $5 \mu \mathrm{L}$ of EDTA $0.5 \mathrm{M}$ and plates incubated overnight at $37^{\circ} \mathrm{C}$. Any clear extension of the inhibition zone around the carbapenem disk toward the EDTA disk was interpreted as a positive result. In the prior method, Modified Hodge test, that is recommended by CLSI, plate surface inoculated by E. coli ATCC 25922. An imipenem disk placed in the center and A. baumannii isolates inoculate the plate in a line from edge toward center, near the imipenem disk. Plates incubated overnight in $37^{\circ} \mathrm{C}$. Any bending at the crossing line of $E$. coli inhibition zone and isolates considered as positive result. A self-collection VIM producer Pseudomonas aeruginosa was used as positive control.

\section{Results}

Bacterial isolates: As shown in Table 1, 33 out of 40 (82.5\%) of A. baumannii are isolated from tracheal aspirates. Other sources are close in frequency. Table 2 shows the frequency of $A$. baumannii in regard to hospital wards. As expected, most of isolates belong to ICU, which show the opportunistic potential of this pathogen.

Antibacterial susceptibility testing: Selected antibiotics for antibiogram testing were based on common treatment $[13,14]$. Intermediate susceptibility was considered as resistant. Table 3 shows the rate of resistance in our clinical isolates. Carbapenems are still the most effective agents against multidrug resistant A. baumannii. Cephalosporins have no effect in susceptibility testing. Aminoglycosides, tobramycin in case, may have better therapeutic effects than cephalosporins. Colistin has as the same as result of cephalosporins, but however, disk diffusion is not recommended by CLSI for this antibiotic [11].

Phenotypic detection of MBLs: Twenty three out of 40 isolates (57.5\%) were carbapenem resistant A. baumannii (CRAB) by the disk diffusion method. Phenotypic screening for MBL producers were done by 2 methods (MHT and DDT) (Figures 1 and 2). None of isolates show MBL producer's characteristics.

\begin{tabular}{lcccccc}
\hline Table 1. Type of Specimens and Related Frequency \\
\hline Specimen & $\begin{array}{l}\text { Tracheal } \\
\text { Culture }\end{array}$ & $\begin{array}{c}\text { Urine } \\
\text { Culture }\end{array}$ & $\begin{array}{c}\text { Wound } \\
\text { Culture }\end{array}$ & $\begin{array}{c}\text { Blood } \\
\text { Culture }\end{array}$ & $\begin{array}{c}\text { Cerebral Spinal } \\
\text { Fluid }\end{array}$ & $\begin{array}{c}\text { Infection Control } \\
\text { Isolates }\end{array}$ \\
\hline No. $(\%)$ & $33(82.5)$ & $2(5)$ & $1(2.5)$ & $1(2.5)$ & $1(2.5)$ & $2(5)$ \\
\hline
\end{tabular}

Table 2. Hospital Wards and Number of Referred Specimens

\begin{tabular}{lccccc}
\hline Ward & ICU & Outpatient & Orthopedics & Internal & Burn \\
\hline No. $(\%)$ & $35(87.5)$ & $2(5)$ & $1(2.5)$ & $1(2.5)$ & $1(2.5)$ \\
\hline
\end{tabular}


Table 3. Antibiotic Susceptibility of Acinetobacter baumanii Isolates $\mathrm{a}, \mathrm{b}$

\begin{tabular}{lcc}
\hline & Susceptible & Resistant \\
\hline IMI & $27(67.5)$ & $13(22.5)$ \\
\hline MEM & $17(42.5)$ & $23(57.5)$ \\
\hline CAZ & $3(7.5)$ & $37(92.5)$ \\
\hline CTX & $2(5)$ & $38(95)$ \\
\hline CRO & $2(5)$ & $38(95)$ \\
\hline CPM & $2(5)$ & $38(95)$ \\
\hline PRL & $2(5)$ & $38(95)$ \\
\hline ATM & $2(5)$ & $38(95)$ \\
\hline AMP/SUL & $4(10)$ & $36(90)$ \\
\hline AK & $3(7.5)$ & $37(92.5)$ \\
\hline TOB & $11(27.5)$ & $29(72.5)$ \\
\hline GM & $3(7.5)$ & $37(92.5)$ \\
\hline CIP & $4(10)$ & $36(90)$ \\
\hline COL & $2(5)$ & $38(95)$ \\
\hline A & $; A 45 U L$ & \\
\hline
\end{tabular}

a Abbreviations: AK, amikacin; AMP/SUL, ampicillin-sulbactam; ATM, aztreonam; CAZ, ceftazidime; CIP, ciprofloxacin; CRO, ceftriaxone; COL, Colistin; CPM, cefepime; CTX, cefotaxime; GM, gentamicin; IMI, imipenem; MEM, meropenem; PRL, piperacillin;; TOB, tobramycin. b Data are presented as No. (\%).

Figure 1. Modified Hodge Test

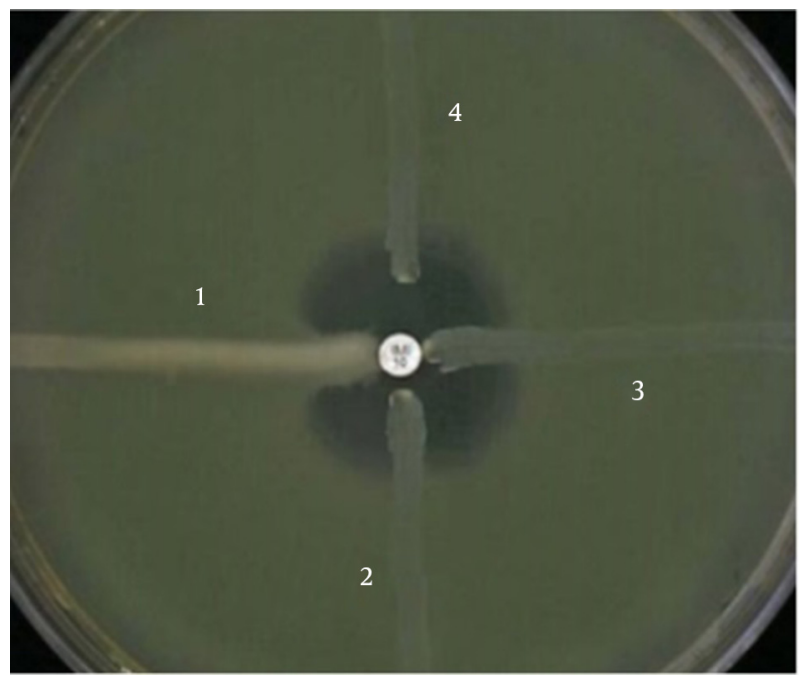

Line 1 is VIM positive $P$. aeruginos $a$, as positive control. Lines 2,3 and 4 are clinical carbapenem resistant $A$. baumannii isolates. The background of MHB is inoculated by E. coli ATCC 25922.
Figure 2. Double Disk Test With Negative Result of non-MBL A. baumannii Isolate

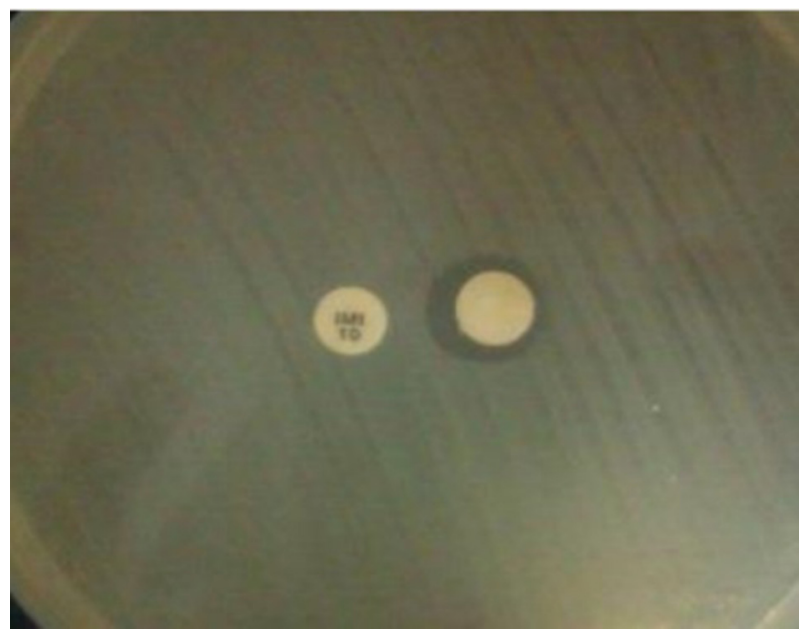

Blank disk (right) is moistened by $5 \mu \mathrm{L}$ of 0.5 M EDTA. Disks are arranged in $1 \mathrm{~cm}$ edge to edge.

\section{Discussion}

In this study, antibiotic profile and phenotypic detection of A. baumannii isolates were sought. Susceptibility testing showed high level of resistance in our clinical setting, especially those related to cephalosporins as well as aminoglycosides. Carbapenems had shown the best efficacy against $A$. baumannii isolates. In the one hand there are highly carbapenem resistant isolates (57.5\%), but in the other hand no MBL producer isolate detected. These findings are in accordance with previously reported data from Afzalipur hospital [15] and elsewhere [16, 17]. A report by Asadollahi et al. in burn patient from Tehran revealed complete susceptibility to colistin [18].

More recently, new methods for phenotypic detecting carbapenemase producing isolates have been described based on expansion of inhibition zone [19, 20]. They have used other materials like mercaptoacetate and dipicolinic acid which shows statistical and practical benefits. However, these methods require more studies to be accepted by laboratories.

Among aminoglycoside, tobramycin had better results than gentamicin and amikacin. aminoglycoside modifying enzymes are widely distributed in A. baumannii isolates [21]. A molecular method such as PCR is required to seek these enzymes. Besides such enzymes, outer membrane proteins are involved in resistance to aminoglycosides.

According to Table 1, most of isolates are from tracheal specimens. The dominant way for spreading bacteria in hospitals is from hands of staffs. Physical operation in tracheal catheterization is the potential way for transferring Acinetobacter spp. to the hospitalized patients. Hand washing is recommended by the experts to control such 
spreading [22, 23]. Effective hospital programs to control healthcare-associated infections improve treatments of patients further to decrease the risk of spreading opportunistic bacteria [23]. Besides, economical costs are of concern for antimicrobial resistant infections, which should be considered as hospital priority.

Nearly all of isolates were resistant to colistin, which was not our expectance. For colistin susceptibility evaluation CLSI recommends minimum inhibitory concentration (MIC) as standard method, however, according to funding limitations, this procedure was not available in our hands.

CRAB isolates were totally isolated from ICU patients, mostly from tracheal aspirates, which is in accordance with other reported $[6,24]$. A study by Shahcheraghi et al. showed similar results, however, they found that MBL and ESBL A. baumannii isolates which encode SPM and GES types, respectively, in very few number of isolates [25]. These data show colonization of CRAB isolates in ICU ward. Although, underlying diseases were not considered in our study, but the most common underlying condition in patients were venous and tracheal catheterizations. Risk factors for ICU-acquired carbapenem isolates are well-investigated [26]. Surgical operations, previous carbapenem prescription and the time of ICU stay are associated with carbapenem resistance [27]. A typing method is required in this case, which is in progress for future clonal analysis. Although, carbapenem resistant is common phenomenon in A. baumannii isolates, but, MBL enzymes are not frequently responsible in case [7]. As noted previously, carbapenem resistant $A$. baumannii (CRAB) is ascribed to reasons like producing oxacillinases (OXA) and metallo beta lactamases (MBLs), efficient efflux pumps and in parts, reduced expression of outer membrane proteins $[7,8]$.

In conclusion, carbapenem resistance is not due to MBL enzymes in our clinical setting. More investigations are required to understand resistance profile and clonality pattern of A. baumannii isolates.

\section{Acknowledgements}

The authors would thank Afzalipur laboratory staff for their valuable assistance.

\section{Authors' Contributions}

Conceived and designed the experiments: Himen Salimizand, Omid Azizi, Farzan Modarresi, Shahla Mansouri. Performed the experiments: Himen Salimizand, Omid Azizi, Farzan Modarresi, Mehdi Rahmati. Analyzed the data: Himen Salimizand. Contributed reagents/materials/ analysis tools: Himen Salimizand, Omid Azizi, Farzan Modarresi. Wrote the paper: Himen Salimizand.

\section{Funding/Support}

Kerman University of Medical Sciences, Student Research Committee, code 91/155.

\section{References}

1. Yamamoto M, Nagao M, Matsumura Y, Hotta G, Matsushima A, Ito $\mathrm{Y}$, et al. Regional dissemination of Acinetobacter species harbouring metallo-beta-lactamase genes in Japan. Clin Microbio Infect. 2013;19(8):729-36.

2. Mezzatesta ML, D'Andrea MM, Migliavacca R, Giani T, Gona F, Nucleo E, et al. Epidemiological characterization and distribution of carbapenem-resistant Acinetobacter baumannii clinical isolates in Italy. Clin Microbiol Infect. 2012;18(2):160-6.

3. Weisenberg SA, Schuetz AN, Alexander EL, Eiss B, Behta M, Saiman $\mathrm{L}$, et al. Endemic Acinetobacter baumannii in a New York hospital. PLoS One. 2011;6(12):e28566.

4. Azim A, Dwivedi M, Rao PB, Baronia AK, Singh RK, Prasad KN, et al. Epidemiology of bacterial colonization at intensive care unit admission with emphasis on extended-spectrum beta-lactamaseand metallo-beta-lactamase-producing Gram-negative bacteria-an Indian experience. J Med Microbiol. 2010;59(Pt 8):955-60.

5. Sengstock DM, Thyagarajan R, Apalara J, Mira A, Chopra T, Kaye KS. Multidrug-resistant Acinetobacter baumannii: an emerging pathogen among older adults in community hospitals and nursing homes. Clin Infect Dis. 2010;50(12):1611-6.

6. Li J, Rayner CR, Nation RL, Owen RJ, Spelman D, Tan KE, et al. Heteroresistance to colistin in multidrug-resistant Acinetobacter baumannii. Antimicrob Agents Chemother. 2006;50(9):2946-50.

7. Higgins PG, Dammhayn C, Hackel M, Seifert H. Global spread of carbapenem-resistant Acinetobacter baumannii. J Antimicrob Chemother. 2010;65(2):233-8.

8. Sinha M, Srinivasa H. Mechanisms of resistance to carbapenems in meropenem- resistant Acinetobacter isolates from clinica samples. Indian J Med Microbiol. 2007;25(2):121-5.

9. Bonomo RA, Szabo D. Mechanisms of multidrug resistance in Acinetobacter species and Pseudomonas aeruginosa. Clin Infect Dis. 2006;43 Suppl 2:S49-56.

10. Walsh TR, Toleman MA, Poirel L, Nordmann P. Metallo-betalactamases: the quiet before the storm? Clin Microbiol Rev. 2005;18(2):306-25.

11. Clinical and Laboratory Standards Institute. Clinical and Laboratory Standards Institute Performance standards for antimicrobial susceptibility testing Twenty-Second Informational Supplement M100-S22.Wayne, PA, USA: CLSI; 2012.

12. Kim SY, Hong SG, Moland ES, Thomson KS. Convenient test using a combination of chelating agents for detection of metallo-beta-lactamases in the clinical laboratory. J Clin Microbiol. 2007;45(9):2798-801.

13. Yan JJ, Wu JJ, Tsai SH, Chuang CL. Comparison of the double-disk, combined disk, and Etest methods for detecting metallo-betalactamases in gram-negative bacilli. Diagn Microbiol Infect Dis 2004;49(1):5-11.

14. Mandell GL, Bennett JE, Dolin R. Principles and practice of infetiou diseases. 6th ed, Philadelphia: Churchill Livingstone; 2004

15. Shakibaie MR, Adeli S, Salehi MH. Antibiotic resistance patterns and extended-spectrum beta-lactamase production among Acinetobacter spp. isolated from an intensive care Unit of a hospital in Kerman, Iran. Antimicrob Resist Infect Control. 2012;1(1):1.

16. Peymani A, Farajnia S, Nahaei MR, Sohrabi N, Abbasi L, Ansarin K, et al. Prevalence of class 1 integron among multidrug-resistant Acinetobacter baumannii in Tabriz, northwest of Iran. Pol J Microbiol. 2012;61(1):57-60.

17. Peymani A, Nahaei MR, Farajnia S, Hasani A, Mirsalehian A, Sohrabi N, et al. High prevalence of metallo-beta-lactamase-producing acinetobacter baumannii in a teaching hospital in Tabriz, Iran. Jpn J Infect Dis. 2011;64(1):69-71.

18. Asadollahi P, Akbari M, Soroush S, Taherikalani M, Asadollahi K, Sayehmiri K, et al. Antimicrobial resistance patterns and their encoding genes among Acinetobacter baumannii strains isolated from burned patients. Burns. 2012;38(8):1198-203.

19. Wachino J, Matsui M, Tran HH, Suzuki M, Suzuki S, Shibayama K Evaluation of a double-disk synergy test with a common metallobeta-lactamase inhibitor, mercaptoacetate, for detecting NDM1-producing Enterobacteriaceae and Acinetobacter baumannii. Jpn J Infect Dis. 2014;67(1):66-8.

20. Yong D, Lee Y, Jeong SH, Lee K, Chong Y. Evaluation of double- 


\section{Salimizand H et al.}

disk potentiation and disk potentiation tests using dipicolinic acid for detection of metallo-beta-lactamase-producing pseudomonas spp. and Acinetobacter spp. J Clin Microbiol. 2012;50(10):3227-32.

21. World Health Origanization. Guidelines on hand hygiene in health care - global patient safety challenge 2005-2006. Available from: http://www.who.int/patientsafety/en/.

22. Siegel JD, Rhinehart E, Jackson M, Chiarello L. Management of multidrug-resistant organisms in health care settings, 2006. Am J Infect Control. 2007;35(10):S165-93.

23. Pittet D, Hugonnet S, Harbarth S, Mourouga P, Sauvan V, Touveneau $S$, et al. Effectiveness of a hospital-wide programme to improve compliance with hand hygiene. Infection Control Programme. Lancet. 2000;356(9238):1307-12.
24. Lee K, Yong D, Jeong SH, Chong Y. Multidrug-resistant Acinetobacter spp.: increasingly problematic nosocomial pathogens. Yonsei Med J. 2011;52(6):879-91.

25. Shahcheraghi F, Abbasalipour M, Feizabadi M, Ebrahimipour $\mathrm{G}$, Akbari N. Isolation and genetic characterization of metallobeta-lactamase and carbapenamase producing strains of Acinetobacter baumannii from patients at Tehran hospitals. Iran J Microbiol. 2011;3(2):68-74

26. Harris AD, Smith D, Johnson JA, Bradham DD, Roghmann MC. Risk factors for imipenem-resistant Pseudomonas aeruginosa among hospitalized patients. Clin Infect Dis. 2002;34(3):340-5.

27. Akinci E, Colpan A, Bodur H, Balaban N, Erbay A. Risk factors for ICU-acquired imipenem-resistant Gram-negative bacterial infections. J Hosp Infect. 2005;59(4):317-23. 